\section{Draft Mental Health Bill in England}

Dr Maden's commentary on...'The Draft Mental Health Bill in England: without principles' (Psychiatric Bulletin, July 2005, 29, 250-251) is dismissive of 'liberal hysteria' and suggests that therapeutic intent is a peripheral issue to use of mental health law. The perspective of someone viewing the issues from a tertiary service probably explains the failure to understand the ramifications that the proposed Bill will have on the population as a whole. The ability of general adult services to select in-patients on the basis of need or evidence of effectiveness would be paralysed. General psychiatric hospitals will return to the dark days of having a primary social control function. Apart from the ethical considerations of using a hospital as a prison, does anyone really believe this legislation will lead to better protection of the public? We are looking in the wrong legislative direction for solutions to this.

Dr Maden's commentary concludes by suggesting that our current mental health legislation is among the best and most liberal in the world in the way in which it deals with offenders with mental illness. Why on earth, then, are we proposing to change it?

Martin Gee Consultant Adult Psychiatrist, Ashcombe Centre, Wall LaneTerrace, Cheddleton, Staffordshire ST13 7ED

\section{Electronic care record}

Dr Holloway describes the moves to introduce an electronic care record (ECR) (Psychiatric Bulletin, July 2005, 29, 241243). He raises concerns that 'important qualitative aspects' may be lost in the transition from existing medical records.

There is no reason to suppose that this should be the case. There is nothing contained in traditional records that cannot be easily translated to electronic form. The 'qualitative aspects' may be contained in free text notes or diagrams, and technology to include these is readily available. In addition, the fact that the record will be permanently accessible nationwide (and clearly legible) may encourage fuller and more informative recording than at present.

Clearly, the mechanism of entry will change from pen and paper to keyboard and mouse. This will pose no problem to the many increasingly IT-literate trainees, and for some will make data entry faster and more accurate. For everyone else, emergent technologies such as voice recognition may be appropriate or the secretarial role could be expanded to include typing of entries. Many documents (out-patient letters, minutes of meetings, etc) are already typed and held on computer, so including these in the ECR should be straightforward. There are clearly resource implications, but there will also be savings, as many labour-intensive aspects of paper notes (fetching and carrying, filing, locating records, etc) will no longer be needed.

Many trusts have already introduced some form of electronic patient record with success. None of the problems posed is insuperable, and with appropriate planning the ECR should surpass traditional medical records in every aspect.

J. D. Reed Senior House Officer in Psychiatry of Learning Disability, Heath Lane Hospital, West Bromwich, West Midlands B71 2BG

\section{Do we need a wider survey of physical healthcare provision for psychiatric patients?}

Very few of us, I suspect, will have been surprised to read that there is inadequate recording of physical health parameters in psychiatric notes, but we should still be disappointed to learn of Dr Greening's findings (Psychiatric Bulletin, June 2005, 29, 210-212). However, in failing to reflect upon the contribution of other professionals I am concerned that this study invites a distorted view of physical healthcare provision as a whole, which surely should be the main issue for our patients.

All psychiatric patients should be encouraged to register for and utilise primary care services, and this is especially so for rehabilitation for patients for who it is part of returning to a 'normal' way of life. When working in rehabilitation psychiatry, the prevailing attitude was that it is appropriate and non-discriminatory for our patients to take some of their physical complaints to general practitioners who see these presentations regularly. Sometimes (probably not often enough) we would be informed of these consultations by letter, but even then doubt whether very many of us would copy this information into the handwritten notes. I am concerned that by only looking at secondary care case notes this survey would not have adequately detected input from primary care.

Auditing against pronouncements from the National Institute for Clinical Excellence has a certain kind of validity, but perhaps the salient question here is 'how is the physical health of our patients recorded in its entirety?' The new general medical services contract explicitly states that primary care is responsible for providing physical healthcare to people with serious mental illness (Lester, 2005). I am worried that in omitting mention of primary care's contribution in any part of the discussion this paper invites us to conclude that these findings represent the full extent to which the physical health of psychiatric patients is recorded by those who are responsible for doing so. I feel this is potentially misleading.

LESTER, H. (2005) Shared care for people with mental illness: a GP's perspective. Advances in Psychiatric Treatment, 11, 133-139.

Andy Bickle Senior House Officer in Forensic Psychiatry, Rampton Hospital, Retford, Nottinghamshire DN22 OPD

\section{Cranial computed tomography in old age psychiatry}

I read with interest Dr Fielding's paper on the value of cranial computed tomography in old age psychiatry (Psychiatric Bulletin, January 2005, 29, 21-23). In a similar audit in the old age psychiatry service in southeast Hertfordshire, exploring the role of neuroimaging in the investigation of dementia, of 88 patients, who had undergone computed tomography or magnetic resonance imaging of brain, two were reported to have potentially reversible causes of dementia. One showed a meningioma that was considered to be an incidental finding and not causally related to dementia. The other had disproportionately dilated ventricles, suggestive of normal pressure hydrocephalus. However, this diagnosis was not confirmed on subsequent review. There were 17 patients with other focal abnormalities: 14 showed old infarcts, not suspected from the clinical history in four; two patients had focal frontal atrophy, which was unsuspected in one prior to the scan; one patient had cavum septum pellucidum and basal ganglia calcification. Although the scans led to a revision of the aetiology of dementia in some cases, the impact on subsequent management was not significant. Although this audit was conducted in a smaller sample, its findings are largely in agreement with the results of Dr Fielding.

A. K. Upadhyaya Consultant Psychiatrist for Older Adults and Honorary Senior Lecturer. Herts and Essex Hospital, Cavell Drive, Bishop's Stortford, Herts CM23 5JH

\section{Modernising psychiatric education}

The article by Dr Brown et al (Psychiatric Bulletin June 2005, 29, 228-230) on modernising psychiatric education summarised very well the current position and thinking with regards to the overhaul of medical education and psychiatric education in particular. I doubt though that enough emphasis has been 
given to who is actually going to provide this education. Dr Brown et al allude to potential problems when they say that adequate planning and fair warning has to be given to trainers and trainees about the intended changes, but it remains unclear whether these can be achieved in a climate of continuous recruitment problems in psychiatry. As a senior house officer scheme organiser, trainer and honorary lecturer I am also aware of the time constraints, which already limit the amount of time that consultants can spend with their trainees. Moreover, the new shift systems have significantly reduced the amount of time for consultants and trainees to meet. The proposals, as outlined in the article, emphasise more modular and assessment-based teaching, which in turn will inevitably require much more time devoted to trainees by their trainers. It is absolutely vital that before we embark on such significant changes we make sure that they can actually be delivered on the ground, which I very much doubt is possible with current staffing levels.

P. Lepping Consultant Psychiatrist/Honorary Lecturer, Llwyn Y Groes Psychiatric Unit, North East Wales NHS Trust, Wrexham Maelor Hospital, Croesnewydd Road, Wrexham LL13 7TD, e-mail: PETER.LEPPING@new-tr.wales.nhs.uk

\section{Violence risk assessment}

Dr Maden's editorial (Psychiatric Bulletin, April 2005, 29, 121-122) in response to the article by Higgins et al (Psychiatric Bulletin, April 2005, 29, 131-133) neglects a fundamental aspect of risk assessment and his recommendations therefore need to be treated with caution. Higgins et al refer to the different contexts in which risk assessment forms were being used, but Dr Maden does not appear to have taken this fully into account in advocating the routine use of the Historical/Clinical Risk-20 (HCR-20) in adult general psychiatry.

There is no doubt that the HCR-20 is a useful tool in forensic settings, where it is already widely used. However, there would be significant time and cost implications to introducing it routinely into general adult settings, and it could be argued that this would not be sensible or cost-effective. Although Dr Maden starts by advocating 'a more systemic approach in marginal cases' which it is hard to disagree with, his advocacy of the routine use of the 'ideal' HCR-20 does not appear to address whether this is a feasible option with non-marginal cases. The HCR-20 can be very effective in supporting teams in assessing and reviewing ongoing risks, but it is not clear that it is a practical solution to supporting, for example, the risk assessment and management decisions of a junior psychiatrist doing an assessment of a new patient in an accident and emergency department in the middle of the night.

Dr Maden notes that Higgins et al reported that many consultants did not attend the (presumably free) half-day training on violence risk assessment already on offer within their services. To suggest 3-day external fee-based training specifically for violence risk assessment seems a disproportionate response when other priorities in mental health (for example assessing the risk of self-harm) are not identified as requiring such expensive formalised training.

It is a pity that neither Higgins et al nor Dr Maden were able to expand on the possible utility of the CARDS project (Watts et al, 2004) that the study of Higgins et al was part of. This appears to be a worthwhile collaborative attempt at developing a more standardised approach to risk assessment in general adult psychiatry. It is also free and potentially more easily integrated into routine clinical practice than the wholesale use of the HCR-20.

WATTS, D., BINDMAN, J., SLADE, M., et al (2004) Clinical assessment of risk decision support (CARDS): The development and evaluation of a feasible violence risk assessment for routine psychiatric practice. Journal of Mental Health, 13, 569-581.

Dan Beales Specialist Registrar in Forensic Psychotherapy, The Red House, Bolton, Salford and Trafford Mental Health NHS Trust, 78 Swinton Road, Salford M27 8GB, e-mail: Daniel.Beales@ edenfield.bstmht.nhs.uk

\section{Clinical excellence awards}

Having once more been through the annual awards process of reviewing citations and CVs, within both trust and College systems, we are writing to express our sense of disillusionment and distaste at the whole rigmarole. Not only is it extremely time-consuming for all involved, especially the applicants, but it is intrinsically unreliable and demeaning as a method of enhancing doctors' pay. The changes in the system from $A, B, C$ to clinical excellence awards and various precious metals have been accompanied by a series of 'domains' that overlap remarkably and for which we have yet to see an agreed model criterion. How does one assess whether an individual doctor is 'delivering a high quality service' or 'managing a high quality service'? What is the definition of a high quality service?

A doctor working overtime because of the poor quality of the service that he or she is involved in perhaps should be preferentially rewarded for staying there rather than going to an easier place. Is 'high quality' defined as patient outcome, for example how many patients with depression are cured or surgical operations carried out without complaint or side-effects, or is it because the service is carried out in accordance with the wishes of the trust, strategic health authority or government? Carrying out an audit or introducing a 'modernised' style of service are automatically noted as positive, but continuing to see difficult patients who complain, don't get better and generate 'untoward incidents' may even downgrade you in your chief executive's eyes.

As with the previous system, the busier and more active the doctors are in seeing patients and providing a comprehensive service - usually beyond contracted hours in the case of many general adult psychiatrists - the less likely they are to be able to sit down and fill out the form with sufficient details of committees or working groups attended, papers published or lectures given. What are termed 'clinical excellence awards' are really awards for clerical excellence. Most of the information is entirely impossible to check, particularly since we do not have a routine patient feedback system (as they do in the USA) or any generally valid outcome measures.

However, even after having created time to fill in the forms, the clinician seeking a national award is then faced with a cruel timetable of waiting until November to find out if an award has been offered and, if not, then having to re-scramble the whole application over Christmas. This is a very difficult time for committees to meet in order to comply with the narrow timetable for submission before the end of January. Once the forms are despatched, the processes and decision-making of the higher Advisory Committee on Clinical Excellence Awards committee levels (i.e. beyond trusts and colleges) is so obscure as to be postKafkaesque. Again, it is likely that these committees and assessors have even less knowledge of the realities behind the forms and will be faced by hundreds of CVs. How can they deal with variations in specialty, age, gender and ignorance of the true resources or quality of care in any given trust?

At the local level matters are even more delicate, small amounts of money per point gained contrasting with the substantial demoralisation following rejection. Many trusts have operated an every second year policy, but is this consistent nationwide? Why not only try and pull your weight every other year? The process becomes essentially an alluring form of salary redistribution that conveniently hides a ceiling on salaries, since the chance of progressing nationally, beyond level 8 , remains less than $10 \%$.

Our view is that these embarrassing and essentially uncertain processes should be abandoned and that the consultant pay scale should be extended into areas of 